\section{Determination of cell fate in skeletal muscle following BMP gene transfer by in vivo electroporation}

\author{
Mariko Kawai, ${ }^{1,2,3}$ Yu-Ki Ohmori, ${ }^{4}$ \\ Mai Nishino, ${ }^{4}$ Masayo Yoshida, ${ }^{4}$ \\ Kaori Tabata, ${ }^{4}$ Do-Saku Hirota, ${ }^{4}$ \\ Ayako Ryu-Mon, ${ }^{4}$ \\ Hiromitsu Yamamoto, ${ }^{2}$ Junya Sonobe, ${ }^{2}$ \\ Yo-Hei Kataoka, ${ }^{3}$ Noriko Shiotsu, ${ }^{3}$ \\ Mika Ikegame, ${ }^{3}$ Hiroki Maruyama, ${ }^{5}$ \\ Toshio Yamamoto, ${ }^{3 *}$ Kazuhisa Bessho, ${ }^{2}$ \\ Kiyoshi Ohura ${ }^{1}$ \\ ${ }^{1}$ Department of Pharmacology, Osaka \\ Dental University, Osaka \\ ${ }^{2}$ Department of Oral and Maxillofacial \\ Surgery, Graduate School of Medicine, \\ Kyoto University \\ ${ }^{3}$ Department of Oral Morphology, \\ Okayama University Graduate School of \\ Medicine and Dentistry and \\ Pharmaceutical Sciences \\ ${ }^{4}$ Dental School of Okayama University \\ ${ }^{5}$ Department of Clinical Nephroscience, \\ Niigata University Graduate School of \\ Medicine and Dental Sciences, Niigata, \\ Japan \\ *Current: Professor Emeritus of \\ Okayama University
}

\begin{abstract}
We previously developed a novel method for gene transfer, which combined a non-viral gene expression vector with transcutaneous in vivo electroporation. We applied this method to transfer the bone morphogenetic protein $(B M P)$ gene and induce ectopic bone formation in rat skeletal muscles. At present, it remains unclear which types of cells can differentiate into osteogenic cells after $B M P$ gene transfer by in vivo electroporation. Two types of stem cells in skeletal muscle can differentiate into osteogenic cells: muscle-derived stem cells, and bone marrow-derived stem cells in the blood. In the present study, we transferred the BMP gene into rat skeletal muscles. We then stained tissues for several muscle-derived stem cell markers (e.g., Pax7, M-cadherin), muscle regenerationrelated markers (e.g., Myod1, myogenin), and an inflammatory cell marker (CD68) to follow cell differentiation over time. Our results indicate that, in the absence of BMP, the cell population undergoes muscle regeneration, whereas in its presence, it can
\end{abstract}

differentiate into osteogenic cells. Commitment towards either muscle regeneration or induction of ectopic bone formation appears to occur five to seven days after $B M P$ gene transfer.

\section{Introduction}

Bone morphogenetic protein (BMP) is known to have strong osteogenic potential when the recombinant protein or the $B M P$ gene is transferred into skeletal muscle. ${ }^{1} \mathrm{We}$ previously developed a novel method for gene transfer, based on transcutaneous in vivo electroporation. ${ }^{2}$ We applied this method to transfer the $B M P$ gene and induce ectopic bone formation in skeletal muscle. Our gene transfer system with in vivo electroporation was applied transcutaneously to the calf muscles of rats, so that only the areas to which electrodes were attached were targeted for gene transfer. Electroporation was conducted at $100 \mathrm{~V}$, with eight 50 -ms pulses. This condition did not result in skin burns. ${ }^{2,3}$ In our previous studies, ectopic bone formation was seen to start ten days after BMP gene transfer, as evidenced by intramembranous or endochondral ossification being restricted to the targeted areas of skeletal muscle ${ }^{3}$. Another gene transfer system expressing the $B M P-2$ gene from an adenoviral vector induced the formation of ectopic bone in nearly all calf muscles. ${ }^{4}$ Therefore, the $B M P-2$ gene transfer system with in vivo electroporation could induce both ectopic bone formation and muscle regeneration in calf muscles. ${ }^{2,3}$

These results revealed that commitment to osteogenic differentiation was determined within ten days of $B M P$ gene transfer and consequent muscle regeneration. At present, it is still unclear which cell types can differentiate into osteogenic cells and cause ectopic bone formation following $B M P$ gene transfer by in vivo electroporation in skeletal muscle.

Skeletal muscle has high potential for self-regeneration. If the muscle is damaged following mechanical injury or toxic insult, satellite cells step in to repair damaged muscle fibres. ${ }^{5}$ Two types of stem cells with the potential to differentiate into osteogenic cells are present in skeletal muscle. ${ }^{6-10} \mathrm{On}$ one hand, skeletal muscles harbour several muscle-derived stem-like satellite cells, which can differentiate into muscle fibres, osteogenic cells, and adipocytes. ${ }^{6-9}$ On the other hand, bone marrow-derived peripheral blood cells are recruited to skeletal muscles via blood flow, thereby differentiating into muscle fibres and osteogenic cells. ${ }^{10}$

In this study, we focused on the muscle-
Correspondence: Dr. Mariko Kawai, Department of Pharmacology, Osaka Dental University, 8-1 Kuzuhahanazono-cho, Hirakata city, Osaka 573-1121, Japan. Tel. +81.72.8643280 - Fax: +81.72 .8643283 . E-mail:kawai-m@cc.osaka-dent.ac.jp

Key words: Cell fate; skeletal muscle; BMP; gene transfer; in vivo electroporation.

Contributions: MK, experiments conception and design; YKO, MN, MY, KT, DSH, ARM, YHK, NS, MI, HY, immunohistochemical analyses performing; MK, immunohistological experiments performing and data analysis; MK, JS, KB, HM, TY, KO, manuscript drafting.

Conflict of interest: the authors declare no conflict of interest.

Received for publication: 13 January 2017 Accepted for publication: 8 March 2017.

This work is licensed under a Creative Commons Attribution-NonCommercial 4.0 International License (CC BY-NC 4.0).

CC Copyright M. Kawai et al., 2017

Licensee PAGEPress, Italy

European Journal of Histochemistry 2017; 61:2772 doi:10.4081/ejh.2017.2772

derived stem cell population responsible for directing muscle regeneration or bone induction after $B M P$ gene transfer by in vivo electroporation. We tried to determine how and when these cells are committed to osteogenic differentiation as opposed to muscle regeneration by comparing them to skeletal muscle electroporated with an empty plasmid.

\section{Materials and Methods}

\section{$B M P$-2 gene transfer to skeletal} muscles of rats using in vivo electroporation

Nine-week-old male Wistar rats $(\mathrm{n}=3$ per group) were purchased from Shimizu Experimental Animal (Kyoto, Japan) and maintained under specific pathogen-free conditions in our animal facility. Rats were anesthetised by intraperitoneal injection of sodium pentobarbital $(5.0 \mathrm{mg}$ per $100 \mathrm{~g}$ of body weight). The fur on the target area of the leg was removed with clippers. We injected the $B M P-2$ gene expression vector, pCAGGS-BMP-2 $(0.5 \mu \mathrm{g} / \mu \mathrm{L})$, at the centre of the calf muscle and immediately electroporated at $100 \mathrm{~V}$ with eight $50-\mathrm{ms}$ pulses 
(Gene Pulser ${ }^{\circledR}$, Ohta Inc., Okayama, Japan). The control group was injected with the empty vector, pCAGGS $(0.5 \mu \mathrm{g} / \mu \mathrm{L})$, under the same conditions as above. All rat procedures were performed in accordance with Okayama University guidelines (OKU2015-137).

\section{Immunohistochemistry}

We observed time course sections one, three, five, and seven days after electroporation with the human $B M P-2$ gene or empty vector. We then stained the sections with haematoxylin-eosin and several muscle-derived stem cell markers, such as paired box 7 (Pax7) and M-cadherin; muscle regeneration markers, such as myogenic differentiation 1 (Myod1) and myogenin; an inflammatory cell marker (CD68); and human $B M P-2$. Anti-Pax7 rabbit polyclonal, anti-Myod1 mouse monoclonal, antimyogenin mouse monoclonal, anti-M-cadherin goat polyclonal, and anti-BMP-2 mouse monoclonal antibodies (all 1:100; Abcam, Cambridge, UK), and anti-CD68 mouse monoclonal antibody (1:50; BioRad, Hercules, CA, USA) were incubated for $12 \mathrm{~h}$ at $4^{\circ} \mathrm{C}$. Sections were then rinsed several times and incubated with a peroxidase-conjugated secondary antibody (1:200; Sigma, St. Louis, MO, USA) for $1 \mathrm{~h}$ at room temperature. Finally, sections were washed with phosphate-buffered saline, immersed in $0.05 \mathrm{M}$ Tris- $\mathrm{HCl}$ buffer $(\mathrm{pH}$ 7.6, $100 \mathrm{~mL}$ ) supplemented with 3,3'diaminobenzidine tetrahydrochloride (20 $\mathrm{mg}$ ) and $30 \% \mathrm{H}_{2} \mathrm{O}_{2}$, and incubated for 10 $\mathrm{min}$ at room temperature.

\section{Results}

\section{Day one after gene transfer}

We observed several haematoxylin-positive cells (Figure 1a, black arrows) as well as damaged or lost muscle fibres (Figure 1a, red arrows) in sections of skeletal muscles transferred with the empty vector. Many CD68-positive cells had appeared in the spaces between muscle fibres (Figure 1b, blue arrows) or had migrated to some of them (Figure 1b, red arrows). M-cadherin could be detected in cells located around damaged muscle fibres (Figure 1c, arrows). Sections of skeletal muscles transferred with the $B M P-2$ gene, were also rich in haematoxylin-positive (Figure $1 \mathrm{~d}, \mathrm{e}$ ) and BMP-2-positive cells (Figure 1f, red arrows). CD68-positive cells exhibited a similar localisation as in muscles transferred with the empty vector (Figure 1g, arrows). Pax7, a marker of satellite stem cells, was detected in some muscle fibres (Figure 1h, arrows).

\section{Day three after gene transfer}

The spaces between muscle fibres appeared enlarged and filled with lymphocyte-like cells showing condensed nuclei (Figure 2 a,c, arrows) following transfer with both empty vector and BMP-2 gene. CD68-positive cells could also be observed following both transfer procedures (Figure 2 b,d). BMP-2-positive cells were found only in the less damaged muscle fibres and were absent from strongly damaged ones (Figure 2e, arrows). Myod1, one of the most important factors in early muscle regeneration, was observed in cells surrounding the spaces between muscle fibres after $B M P-2$ gene transfer (Figure 2f, arrows).

\section{Day five after gene transfer}

Following transfer with the empty vector, we found a spindle-shaped and cytoplasm-enriched cell population in the spaces between muscle fibres (Figure 3a, arrows), as well as a limited number of CD68-positive cells (Figure 3b, arrow), suggesting reduced migration of inflammatory cells. Cells in the area surrounding the spindle-shaped cells, were positive for Pax7 (Figure 3c, arrow) and Myod1 (Figure 3d,
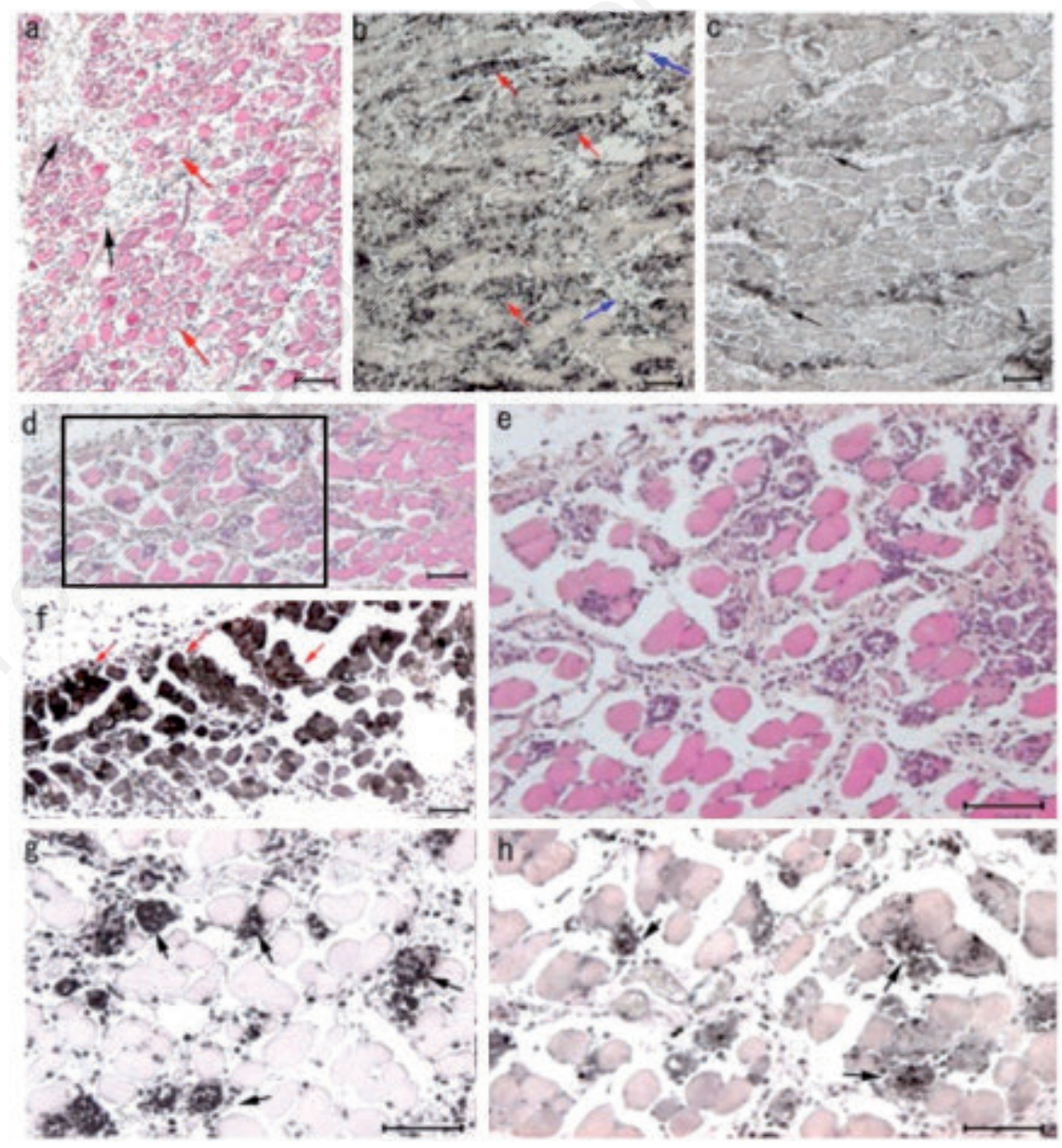

Figure 1. Histological changes in skeletal muscles on day one after empty vector or $B M P-2$ gene transfer. a-c) Skeletal muscles on day one after empty vector transfer. a) Haematoxylin-eosin staining showing migration of inflammatory cells (black arrows) and muscle damage (red arrows). b) CD68-positive cells (red arrows) are detected in the spaces between muscle fibres (blue arrows). c) M-cadherin-positive cells (arrows). d-h) Skeletal muscle one day after BMP-2 gene transfer. d) Haematoxylin-eosin staining. e) Magnification of the insert in (d). f) Human BMP-2-positive cells (red arrows). g) CD68positive cells (arrows). h) Pax7-positive cells (arrows). Scale bars: $100 \mu \mathrm{m}$. 
arrow). Myogenin, a late-stage marker of skeletal muscle regeneration, was detected in cells surrounding the spaces between muscle fibres (Figure 3e, arrows).

In contrast, skeletal muscles transferred with the $B M P-2$ gene presented a canonical elongated muscle fibre appearance, and included haematoxylin-positive cells in the patterned interspaces between muscle lining (Figure 3f). Compared with that at three days after gene transfer, we found fewer CD68-positive cells (Figure 3g). Interestingly, haematoxylin-stained and lymphoid-like cells found in the spaces between muscle fibres were also BMP-2positive (Figure 3h), even though their appearance differed from that on day three. Furthermore, Pax7 (Figure 3i, arrows), Mcadherin (Figure 3j, arrows), and myogenin (Figure 3k, arrows) could be detected around these cells.

\section{Day seven after gene transfer}

In muscles transferred with the empty vector (haematoxylin-eosin staining in Figure 4a), no CD68-positive cells could be detected (Figure 4b), whereas Pax7-positive cells localised around normal muscle fibres (Figure 4c, arrow). In skeletal muscles transferred with the $B M P-2$ gene, cell migration appeared similar to that observed five days after transfer with the empty vector. The cells were spindle-shaped and cytoplasm-enriched (Figure 4d, arrows). Additionally, we could detect a few CD68positive cells in the small spaces between muscle fibres (Figure 4e, arrows). Pax7positive cells were observed in the spindleshaped cell migration area, which also presented abundant extracellular matrix and muscle fibres (Figure 4f, arrows), as well as some alkaline phosphatase-positive cells (Figure 4g, arrows).

\section{Discussion}

In this study, we tried to elucidate what types of cells were recruited and how they migrated when osteogenesis and muscle regeneration occurred simultaneously following in vivo gene transfer with an empty or a BMP-2-expressing vector. To achieve this, we detected cell migration by performing a time course immunohistochemistry analysis of muscle-derived stem cells, inflammation-related cells, and muscle regenerative factors. Muscle-derived satellite stem cells play a crucial role in muscle regeneration, ${ }^{11,12}$ interacting with fibroblasts $^{13}$ and inflammatory cells. ${ }^{14-18} \mathrm{We}$ observed previously that, following vector injection by in vivo electroporation, skeletal muscles exhibited inflammatory reaction and muscle damage. ${ }^{2,3,19,20}$ The inflammatory reaction peaked three days after gene transfer, but terminated by the seventh day. These inflammatory reactions were observed in skeletal muscles not only following transfer with the empty plasmid but also with the $B M P-2$ gene. In the latter, ectopic bone formation was induced on the tenth day after gene transfer, whereby cartilaginous and intramembranous bone tissues were identified within the fibrous tissue that had formed between muscle fibres. ${ }^{3}$ Based on these previous data, we hypothesised that commitment to either osteogenic differentiation or muscle regeneration occurred within seven days after gene transfer, at which point the inflammatory reaction was over. To better understand the timing of the commitment to either fate, we used several histological markers: Pax7, a marker of satellite cells in skeletal muscles; ${ }^{21-23} \mathrm{M}$ cadherin, a marker of mesenchymal stem cells in skeletal muscles; ${ }^{21,24}$ Myod1, a marker of early-stage muscle regeneration; ${ }^{21-24}$ and myogenin, a marker of latestage muscle regeneration ${ }^{21,24}$ plus CD68 as a marker of inflammatory cells. ${ }^{21,25}$

For up to three days, skeletal muscles transferred with either the empty vector or the $B M P-2$ gene presented similar histological profiles, characterised by an ongoing inflammatory reaction. At the same time, M-cadherin-positive mesenchymal stem cells and Pax7-positive satellite cells were seen to migrate to the inflamed areas. Moreover, on the third day, muscle regeneration appeared to be already under way as
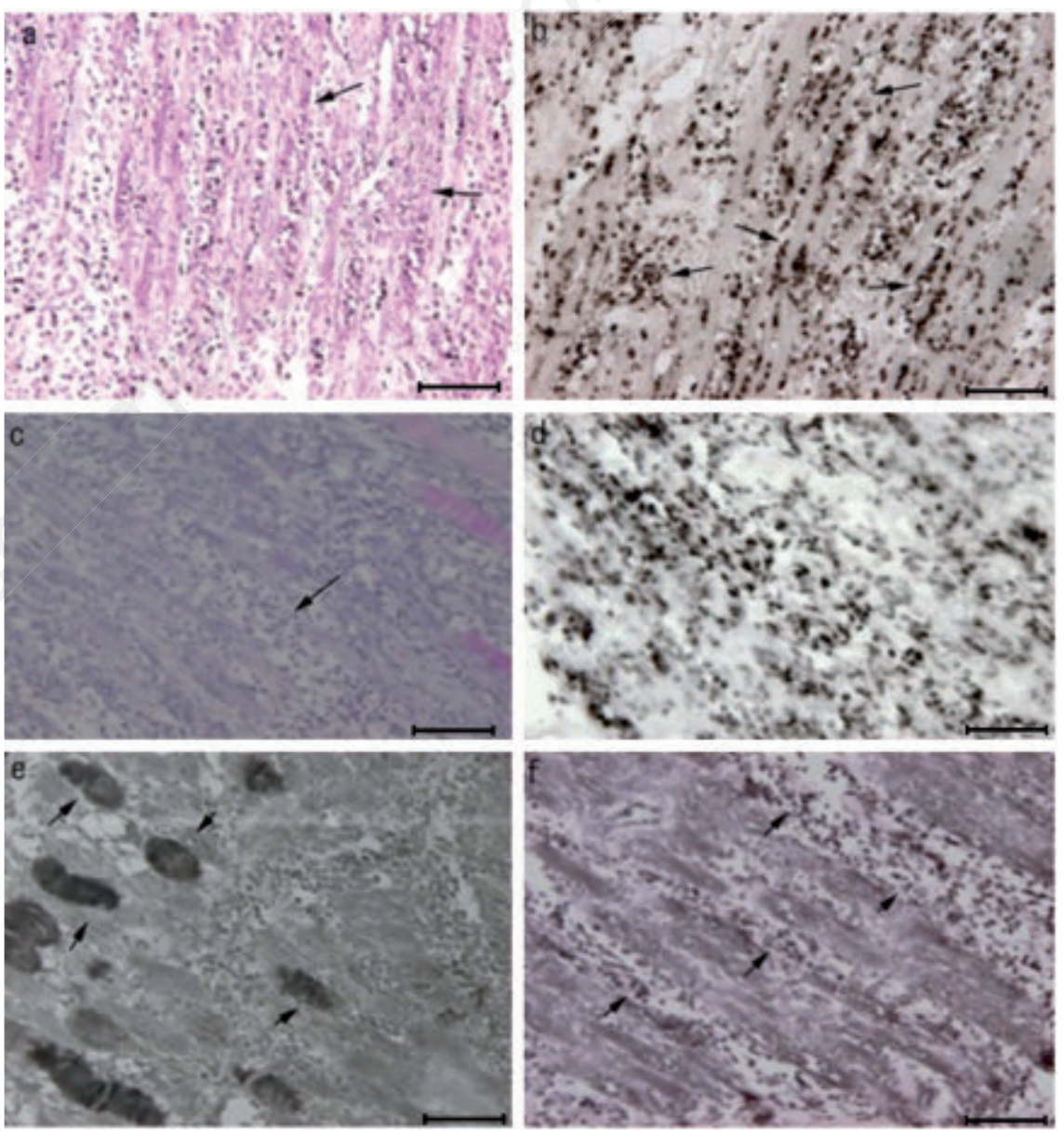

Figure 2. Histological changes in skeletal muscles on day three after empty vector or $B M P-2$ gene transfer. a,b) Skeletal muscles on day three after empty vector transfer. a) Haematoxylin-eosin staining showing enlarged muscle fibres filled with lymphocyte-like cells (arrows). b) CD68-positive cells (arrows). c-f) Histological changes on day three after $B M P-2$ gene transfer. c) Haematoxylin-eosin staining showing enlarged spaces between fibres (arrow). d) CD68-positive cells. e) BMP-2-positive cells are detected only in less damaged muscles (arrows). f) Myod1-positive cells localised around the spaces between muscle fibres (arrows). Scale bars: $100 \mu \mathrm{m}$. 
evidenced by Myod1-positive cells in the inflamed areas. On the fifth day, a population of spindle shaped and cytoplasmenriched cells was observed in the spaces between skeletal muscles. These cells were Pax7- or Myod1-positive, indicating their involvement in skeletal muscle regeneration. We did not find these cells in the interspaces of skeletal muscles transferred with the $B M P-2$ gene; however, we could still detect some CD68-positive cells and several haematoxylin-stained lymphoblast-like cells. Moreover, these lymphoblast-like cells were also BMP-2 positive. For up to three days, BMP-2 positive cells were barely detected in less damaged muscle fibres. On the fifth day after gene transfer, muscles treated with the empty vector were in the middle of the regeneration process. Instead, muscles treated with the BMP-2 gene had started to differentiate and triggered the migration of BMP-2-positive cells to the spaces between muscles fibres. On the seventh day, skeletal muscle regeneration was almost complete in muscles transferred with the empty vector. In contrast, in skeletal muscles transferred with the $B M P-2$ gene, we found CD68- and Pax7-positive cell populations, as well as alkaline phosphatise-positive cells. This indicated that these cell populations changed their direction of differentiation, favouring osteogenesis over muscle regeneration.

At first, gene transfer by in vivo electroporation induced an inflammatory reaction, whereby skeletal muscle-derived satellite stem cells migrated and contributed to muscle regeneration. Skeletal muscle regeneration was complete within seven days. In spite of eliciting an inflammatory reaction, $B M P-2$ gene transfer by in vivo electroporation did not result in sufficiently severe damage within the first three days. Thus, on the fifth day, lymphoblast-like BMP-2-positive cells, rather than skeletal muscle cells, could be detected and seen to populate the spaces between muscle fibre linings. At the same time, skeletal muscle regeneration was also seen to progress simultaneously in some skeletal muscle fibres. On the seventh day, a spindleshaped and cytoplasm-enriched cell population was observed in the interspace of skeletal muscle fibres, together with extracellular matrix containing fibrous tissue. Moreover, alkaline phosphatase-positive cells and satellite cells could be detected in these skeletal muscles. Therefore, induction of ectopic bone formation in skeletal muscles using a $B M P-2$ gene expression vector and in vivo electroporation depends on the sequential appearance of inflammatory cells, myogenic stem cells, BMP-2- positive cells, and finally osteogenic cells.

Taken together, our results suggest that the period between the fifth to the seventh day after $B M P-2$ gene transfer by in vivo electroporation represents the turning point determining commitment to osteogenesis in skeletal muscles. Moreover, Pax7-positive muscle-derived satellite cells appear to play an important role not only during muscle regeneration but also during osteogenesis in the presence of exogenous $B M P-2$. Future studies using the present gene transfer sys-
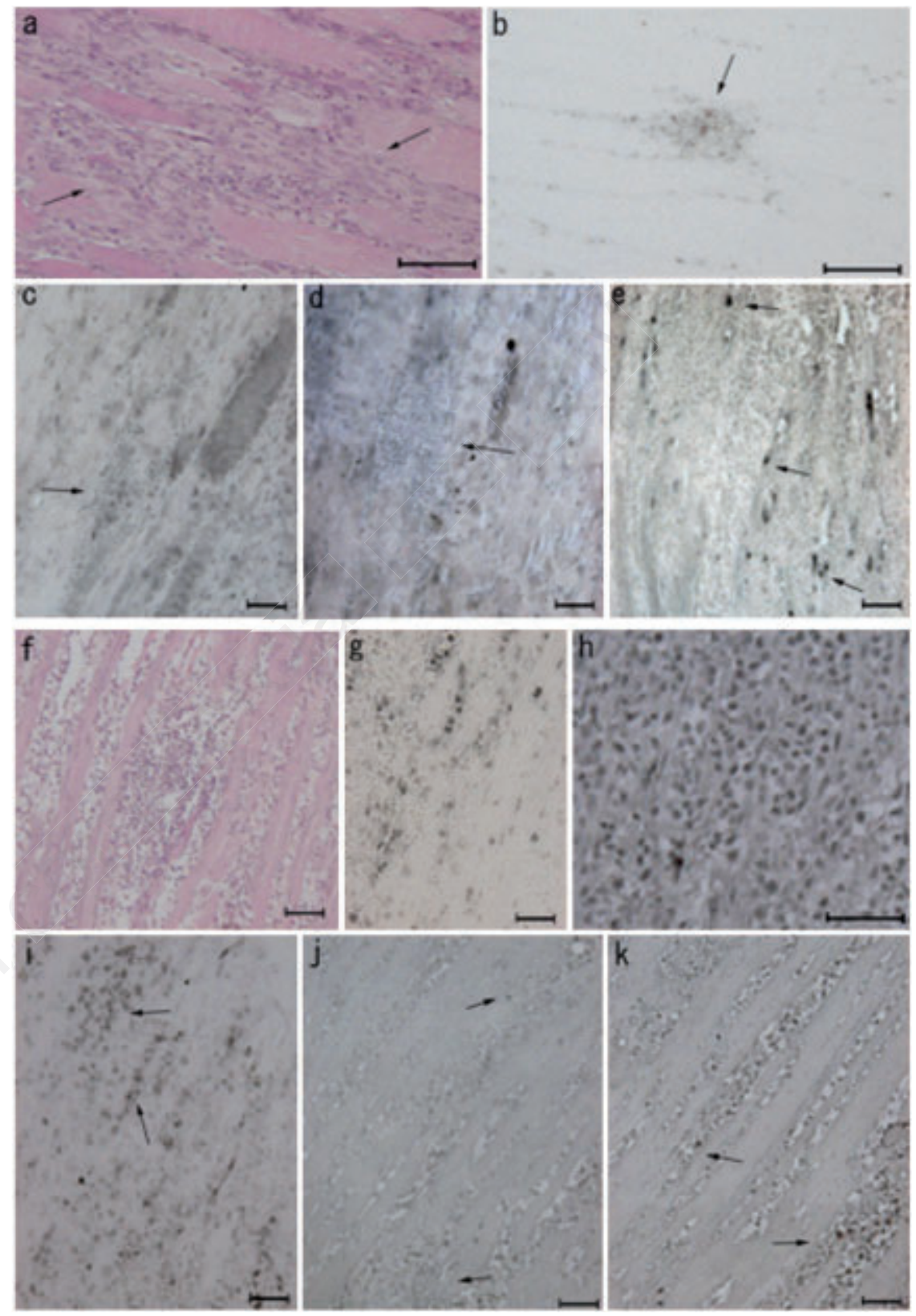

Figure 3. Histological changes on day five after empty vector or $B M P-2$ gene transfer. a-e) Skeletal muscles on day five after empty vector transfer. a) Haematoxylin-eosin staining showing spindle-shaped and cytoplasm-enriched cells in the spaces between muscle fibres (arrows). b) CD68-positive cells (arrow). c) Pax7-positive cells (arrow). d) Myod1positive cells (arrow). e) Myogenin-positive cells are detected around the cell migration area (arrows). f-k) Skeletal muscles on day five after $B M P-2$ gene transfer. f) Haematoxylin-eosin staining showing typical muscle fibres. g) CD68-positive cells. h) BMP-2-positive cells are detected in the haematoxylin-positive population, but not in muscle fibres. i) Pax7-positive cells (arrow). j) M-cadherin-positive cells (arrows). k) Myogenin-positive cells (arrows) are detected between muscle fibres. Scale bars: a,b) $100 \mu \mathrm{m}$; c-k) $50 \mu \mathrm{m}$. 

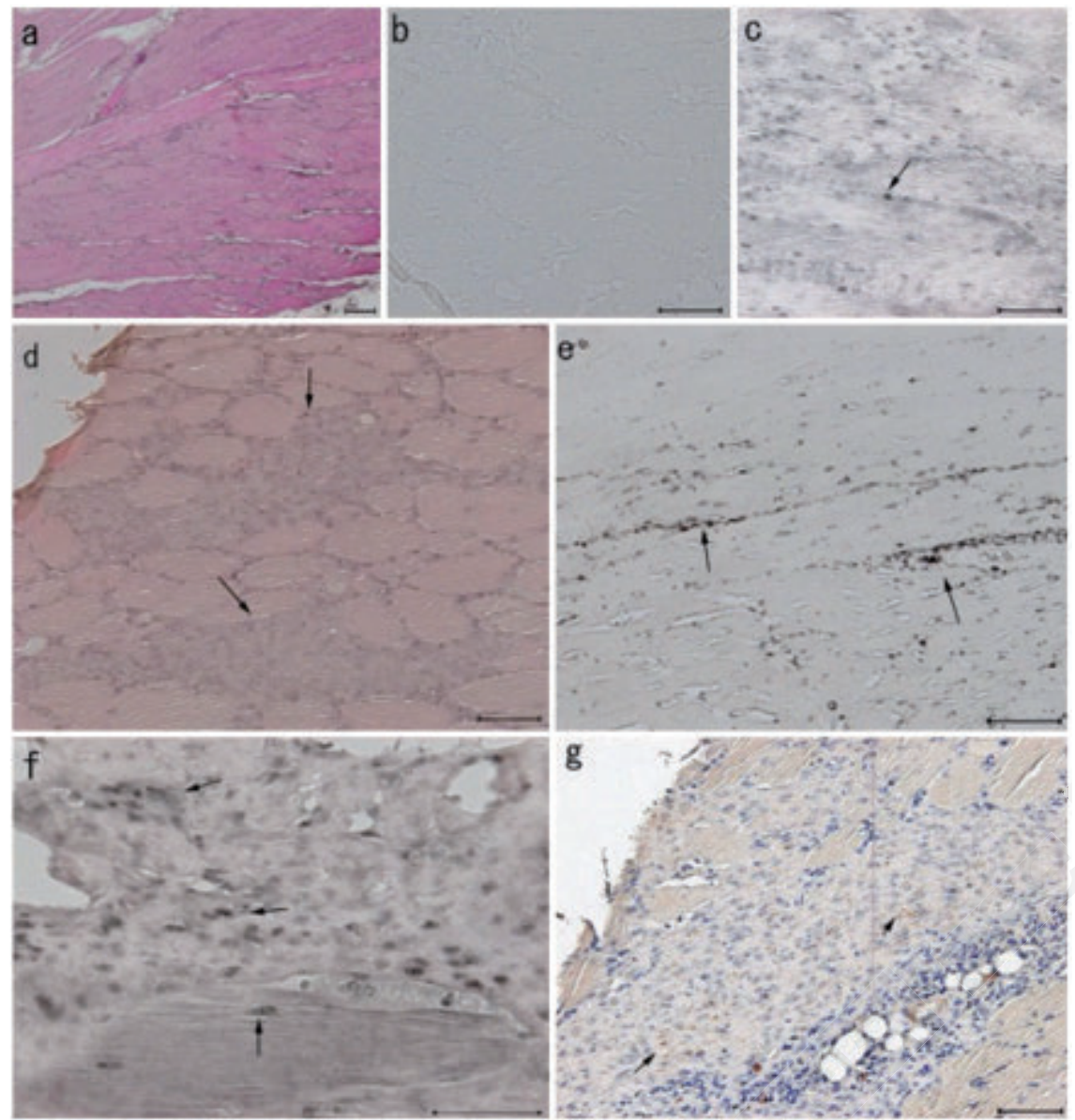

Figure 4. Histological changes on day seven after empty vector or BMP-2 gene transfer. a-c) Skeletal muscles on day seven after empty vector transfer. a) Haematoxylin-eosin staining. b) No CD68-positive cells are detected. c) Pax7-positive cells localised around normal muscle fibres (arrow). d-g) Skeletal muscles on day seven after BMP-2 gene transfer. d) Haematoxylin-eosin staining showing migrating spindle-shaped cells (arrows). e) CD68-positive cells (arrows). f) Pax7-positive cells localised to the spindle-shaped cells migration area (arrows). g) Alkaline phosphatase-positive cells localised to the spindleshaped cell population (arrows). Scale bars: $100 \mu \mathrm{m}$.

tem, should focus on controlling the order defining cell recruitment, migration, and differentiation, as well as the production and control of a meaningful mass of bone for each individual patient.

\section{References}

1. Wang EA, Rosen V, D’Alessandro JS, Bauduy M, Cordes P, Harada T, et al. Recombinant human bone morphogenetic protein induces bone formation. Proc Natl Acad Sci USA 1990; 87:2220-4.

2. Kawai M, Bessho K, Kaihara S, Sonobe J, Oda K, Iizuka T, et al. Ectopic bone formation by human bone morpho- genetic protein-2 gene transfer to skeletal muscle using transcutaneous electroporation. Hum Gene Ther 2003;14: 1547-56.

3. Kawai M, Bessho K, Maruyama H, Miyazaki JI, Yamamoto T. Human BMP-2 gene transfer using transcutaneous in vivo electroporation induced both intramembranous and endochondral ossification. Anat Rec A Discov Mol Cell Evol Biol. 2005;287:1264-71.

4. Kaihara S, Bessho K, Okubo Y, Sonobe J, Kawai M, Iizuka T. Simple and effective osteoinductive gene therapy by local injection of a bone morphogenetic protein-2-expressing recombinant adenoviral vector and FK506 mixture in rats. Gene Ther 2004;11:439-47.

5. Hardy D, Besnard A, Latil M, Jouvion $\mathrm{G}$, Briand D, Thépenier $\mathrm{C}$, et al.
Comparative study of injury models for studying muscle regeneration in mice. PLoS One 2016;11:e0147198.

6. Asakura A, Komaki M, Rudnicki M. Muscle satellite cells are multipotential stem cells that exhibit myogenic, osteogenic, and adipogenic differentiation. Differentiation 2001;68:245-53.

7. Liu R, Birke O, Morse A, Peacock L, Mikulec K, Little DG, et al. Myogenic progenitors contribute to open but not closed fracture repair. BMC Musculoskelet Disord 2011;12:1-9.

8. Cairns DM, Liu R, Sen M, Canner JP, Schindeler A, Little DG, et al. Interplay of Nkx3.2, Sox9 and Pax3 regulates chondrogenic differentiation of muscle progenitor cells. PLoS One 2012;7: e39642.

9. Abou-Khalil R, Yang F, Liu S, Julien A, Perry J, Pereira C, et al. Role of muscle stem cells during skeletal regeneration. Stem Cells 2015;33:1501-11.

10. Lilly MA, Kulkulka NA, Firmiss PR, Ross MJ, Flum AS, Santos GB, et al. The murine bladder supports a population of stromal Sca-1+/CD34+/lin- mesenchymal stem cells. PLoS One 2015;10:e0141437.

11. Lepper C, Partridge TA, Fan CM. An absolute requirement for Pax7-positive satellite cells in acute injury-induced skeletal muscle regeneration. Development 2011;138:3639-46.

12. Sambasivan R, Yao R, Kissenpfennig A, Van Wittenberghe L, Paldi A, GayraudMorel B, et al. Pax7-expressing satellite cells are indispensable for adult skeletal muscle regeneration. Development 2011;138:3647-56

13. Murphy MM, Lawson JA, Mathew SJ, Hutcheson DA, Kardon G. Satellite cells, connective tissue fibroblasts and their interactions are crucial for muscle regeneration. Development 2011;138: 3625-37.

14. Chazaud B, Sonnet C, Lafuste P, Bassez G, Rimaniol AC, Poron F, et al. Satellite cells attract monocytes and use macrophages as a support to escape apoptosis and enhance muscle growth. J Cell Biol 2003;163:1133-43.

15. Gordon S. The macrophage. Bioessays 1995;17:977-86.

16. McLennan IS. Degenerating and regenerating skeletal muscles contain several subpopulations of macrophages with distinct spatial and temporal distributions. J Anat 1996;188:17-28.

17. Grounds MD. Phagocytosis of necrotic muscle in muscle isografts is influenced by the strain, age, and sex of host mice. J Pathol 1987;153:71-82. 
18. Lescaudron L, Peltékian E, FontainePérus J, Paulin D, Zampieri M, Garcia L, et al. Blood borne macrophages are essential for the triggering of muscle regeneration following muscle transplant. Neuromuscul Disord 1999;9:72-80.

19. Kawai M, Bessho K, Maruyama H, Miyazaki JI, Yamamoto T. Simultaneous gene transfer of bone morphogenetic protein (BMP)-2 and BMP-7 by in vivo electroporation induces rapid bone formation and BMP4 expression. BMC Musculoskelet Disord 2006;7:1-11.

20. Yamamoto H, Kawai M, Shiotsu N,
Watanabe M, Yoshida Y, Suzuki K, et al. BMP2 gene transfer under various conditions with in vivo electroporation and bone induction. J Oral Maxillofac Surg Med Pathol 2012; 24:49-53.

21. Ortuste Quiroga HP, Goto K, Zammit PS. Isolation, cryosection and immunostaining of skeletal muscles. Methods Mol Biol 2016;1460:85-100.

22. Tian ZL, Jiang SK, Zhang M, Wang M, Li JY, Zhao R, et al. Detection of satellite cells during skeletal muscle wound healing in rats: time dependent expressions of $\operatorname{Pax} 7$ and MyoD in relation to wound age. Int J Legal Med 2016;130:
163-72.

23. Wu X, Walters TJ, Rathbone CR. Skeletal muscle satellite ell activation following cutaneous burn in rats. Burns 2013;39:736-44.

24. Hill M, Werning A, Goldspring G. Muscle satellite (stem) cell activation during tissue injury and repair. J Anat 2003;203:89-99.

25. Segawa M, Fukada S, Yamamoto Y, Yahagi H, Kanematsu M, Sato M, et al. Suppression of macrophage functions impairs skeletal muscle regeneration with severe fibrosis. Exp Cell Res 2008; 314:3232-44. 